\title{
Efficient Dissemination Technique for Opportunistic Networks
}

\author{
Megha Gupta \\ Department of Information Technology, \\ Jagan Institutes of Management Studies \\ Rohini, Delhi, India
}

\begin{abstract}
Opportunistic Network (OppNet) provides intermittent connectivity in infrastructure less environment without information loss. Intermittent connectivity can be defined as alliance of nodes in a network for communication between them. To establish a routing path in such a heterogeneous environment is a challenging task. The opportunistic networks are also known as Delay Tolerant Network (DTN) as in these types of network, transmission delay associated in relaying data could be very high. In this paper, an efficient data dissemination technique has been introduced to send the information from a Source to Destination. The results generated during simulation for performance metrics such as message delivery ratio, number of data messages generated and average delay are showing the efficiency of the proposed work.
\end{abstract}

\section{Keywords}

Opportunistic Networks, Delay Tolerant Networks, Routing, Flooding.

\section{INTRODUCTION}

Two terminologies OppNet and Delay Tolerant Network (DTN) $[1,2,3]$ are used interchangeably. This type of network concept was taken from Interplanetary Networks (IPN) [4], used for communication between our planet Earth and planet Mars. In OppNet, nodes work on the concept of store-carry and forward mechanism. If a node has data to send, it will forward the data to its neighboring node. In the absence of any neighboring node, the data sending node will buffer the data until it is forwarded to some other nodes. Hence it can be said as waiting for an opportunity to relay the data.

OppNet could be formed using any type of transmission medium e.g. Bluetooth, Infrared rays, Cellular connectivity etc. Nowadays OppNet are used in wireless scenario where the knowledge of source to destination communication link $\mathrm{s}$ not known in advance, or could be unachievable. Military battlefields, natural calamities affected areas; underwater communication, wildlife monitoring, healthcare etc are some of the OppNet's applications areas.

The main advantage of OppNet as compared to other wireless networks is that it can tolerate intermittent connectivity and provides reliable data delivery even with limited knowledge of network. In the traditional networks if the communication link goes down in between of data communication then the data loss is assured. But in OppNet, if the communication link becomes unavailable, even then the data will store in the node's buffer rather being lost. This occurs as in OppNet the message forwarding is done by replicating the message to other nodes.
Main challenges that arise for communication in OppNet are following:-

a. Transmission Delay - Delays may be longer due to unavailability of relay nodes.

b. Energy - If a node's energy finishes before data forwarding, it will result in loss of data and network failure. c. Security - Message delivery occurs through available relay nodes. In this multi-hop communication, there may be the risk of presence of malicious nodes.

d. Storage - As working of OppNet is based on store-carry and forward paradigm, enough storage is required to buffer the data.

The rest of the paper is structured as follows. The next section reviews the related works and briefly describes some of the routing techniques in opportunistic network. Section III describe the proposed model in detail. In Section IV evaluation of the proposed model has been presented. Section $\mathrm{V}$ contains the conclusion and future work.

\section{LITERATURE SURVEY}

In traditional networks, an optimal route is established between source and destination for information flow. But in OppNet, route establishment is done dynamically as per the availability of nodes in the network between source and destination [5,6]. In literature, the available routing protocols for OppNets could be classified as flooding-based and forwarding-based. In flooding based approach, nodes have no prior knowledge of other nodes in the network. It is used for guaranteed delivery in less transmission time. On the other hand, in forwarding based method, nodes have knowledge of other nodes in the network. It is used when resources are limited. Several flooding based routing approaches are discussed in following paragraphs.

In work [7], data is forwarded to the first node which came across. If there are no nodes in the transmission range of the sender, then sender will wait for arrival of any mobile node in its vicinity. In proposed method of [8], source will deliver the message to the destination by itself. If destination is in the transmission range of source, then source will send the data immediately to destination. Else, source will keep waiting for destination to come in its direct contact. Hence the amount of delay incurred may become very high

Epidemic [9] is very simple protocol of OppNet. Whenever any two nodes come in contact, they exchange their buffered message summary with each other. A node will forward those messages that are not available with other nodes. In this way, all the packets will be flooded in the network and reach their destination. This protocol requires high storage capacity on the node and large bandwidth in the network. E.Jones et.al [10] has proposed two-hop relay approach for message 
delivery to the destination. Source node will either deliver directly or through only one intermediate node. Relay node will not further disseminate the message in the network; it will only forward the message to the destination on contact.

T. Small et al [11] have used binary tree based algorithm for flooding, where each node can have maximum of two child nodes only. Source node will generate limited copies to reach certain depth in tree, hence limiting the number of replicas in network. The Spray and Wait [12] algorithm works in two phases named as "Spray phase" \& "Wait phase". In Spray phase, the sender replicates the message to a set of m nodes. These $m$ nodes are selected out of all available neighboring nodes. And these $m$ nodes will further relay the message to other $\mathrm{m}$ relay nodes only. If in spray phase, the destination is not found then the intermediate nodes will store the message and performs direct transmission to the destination.

\section{PROPOSED WORK}

In Epidemic Routing approach the message will be delivered from Source to Destination quickly through flooding. In flooding method, message will be distributed in the whole network that is from Source to intermediate available nodes to Destination. In case of wireless opportunistic networks, node will wait for the next available node in its vicinity to transfer the message, till then it will buffer the message with itself. As shown in figure 1(a), a source, $S$, wishes to reach and deliver its message to a destination, D. But no connected path exists between node $\mathrm{S}$ and node $\mathrm{D}$. Node $\mathrm{D}$ is also not in the range of node S. Hence node S will rely on its neighbor's node for establishing a connection between it and the destination, D. The node $\mathrm{S}$ will transmit its message to nodes $\mathrm{N} 1$ and $\mathrm{N} 2$. After some time, the node N2 is in range of node N5, hence it transmits message to it. Destination 'D' receives message via node $\mathrm{N} 5$ as shown in figure $1(\mathrm{~b})$.

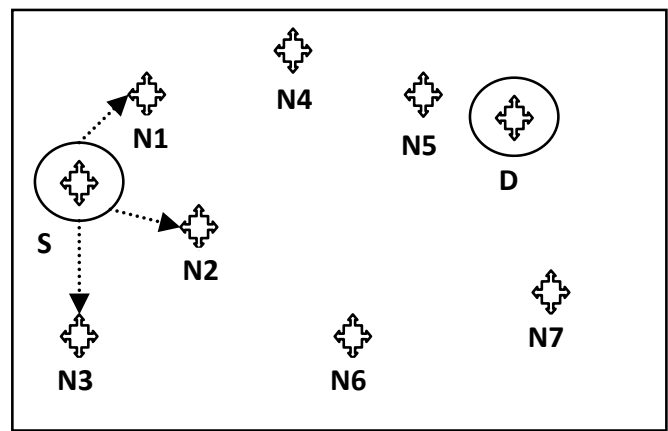

Figure 1(a). Data dissemination in OppNet

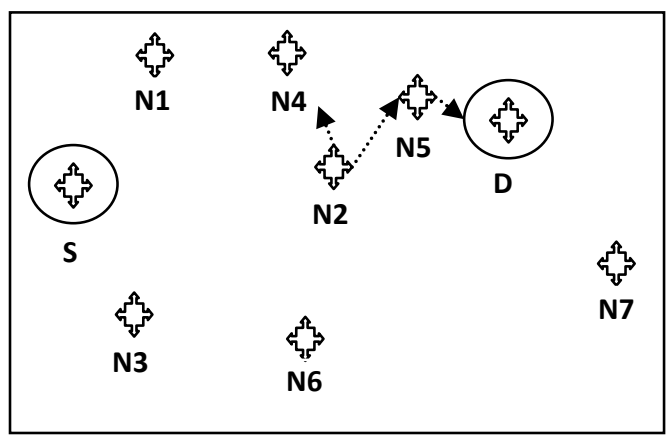

Figure 1(b). Data dissemination in OppNet
In the above approach, if there are more nodes available in the transmission range of the sender node, then all the nodes will receive the data packet to forward to the destination. This approach consumes a lot of available network bandwidth and resources and also may lead to congestion in the network.

In this work selected flooding is performed to provide high reliability. The name of the proposed approach is "ArcBasedSel". In this method, a vector will be created in direction of destination and the nodes will be selected based on their distance against this vector. If in a network, total number of nodes available is $\mathrm{N}$, then out of $\mathrm{N}$ nodes, a set of nodes will participate in flooding of the data packet. It is assumed that the position and direction of destination $\mathrm{D}$ is known. The procedure for selected flooding is as follows:-

1. A directional arc $\vec{A}$ is created from source to destination.

2. A threshold distance parameter $\mathrm{T}$, is set between the node and the directional arc $\vec{A}$.

3. Initially a subset of nodes that are in transmission range of sending node will participate in selection of the forwarding nodes. Distance of the neighboring nodes will be calculated from the directional arc $\vec{A}$.

4. For a node to be member of flooding zone, the distance between node and directional arc $\vec{A}$ should be less than T.

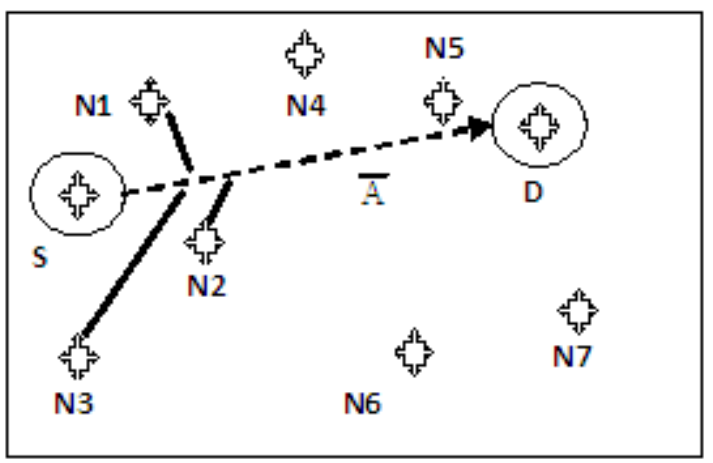

Figure 2. Distance calculation of neighboring nodes

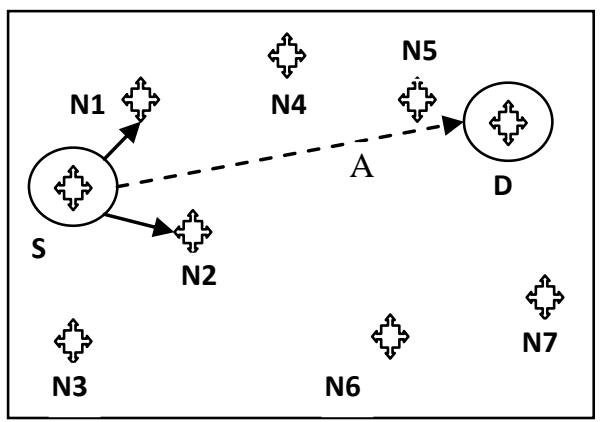

Figure 3. Selection of nodes N1 and N2

As shown in figure 3, nodes N1 and N2 are selected as next relaying nodes. These nodes will again create a directional arc to destination and repeat the below mentioned steps to find next suitable relaying node for data delivery.

Routing Algorithm ArcBasedSel:-

(1) $s \leftarrow$ getSourceID(message)

(2) $d \leftarrow$ getDestinationID(message) 
(3) Create an arc $\bar{A}$ from $s$ to $d$

(4) neighbours $\leftarrow$ s.get Neighbours()

(4) for $m \in$ neighbours do

(5) if distance $(\mathrm{m}, \overline{\mathrm{A}})<\operatorname{distance}(\mathrm{T})$ then

\section{destination}

(10) else

(14) end for setofRelayNode $\leftarrow \mathrm{m}$

for $\mathrm{s} 1 \epsilon$ setofRelayNode do

if $d$ is in range of $s 1$ then complete transmission to

(15)end for

\section{SIMULATION AND ANALYSIS}

Implementation of the proposed selective flooding is done using the ONE[13] which is an Opportunistic Network simulator. The nodes are placed in a rectangular area. Node position is represented with Cartesian $\mathrm{x}$ and $\mathrm{y}$ coordinate system. The proposed work has been compared with epidemic protocol on following parameters:-

- Average Buffer Time - Average time for which message is stored before reaching to destination.

- Delivery Probability- It is taken as percentage of messages successfully delivered to destination.

- Average Transmission Delay - Average delay occur to reach message from source to destination.

Figure 4 shows the effect of number of nodes with respect to average buffer time. It has been observed that the performance of ArcBasedSel is better in comparison of other protocol due to best hop selection from the network. If the best forwarding node is not found then the message is kept in buffer. It is found that average buffer time of ArcBasedSel is $1014 \mathrm{sec}$ which is lesser than that of Epidemic $=1216 \mathrm{sec}$.

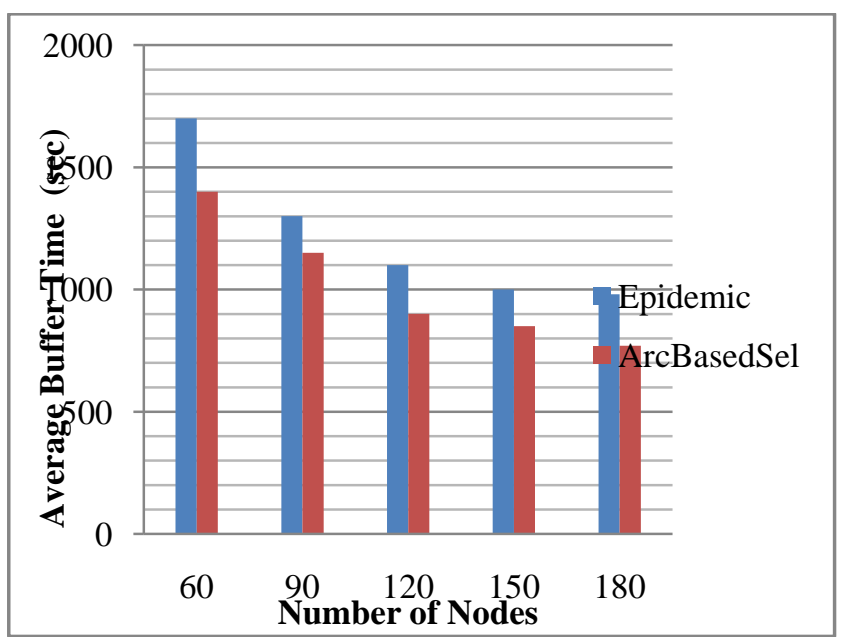

Figure 4. Average Buffer Time vs Number of Nodes

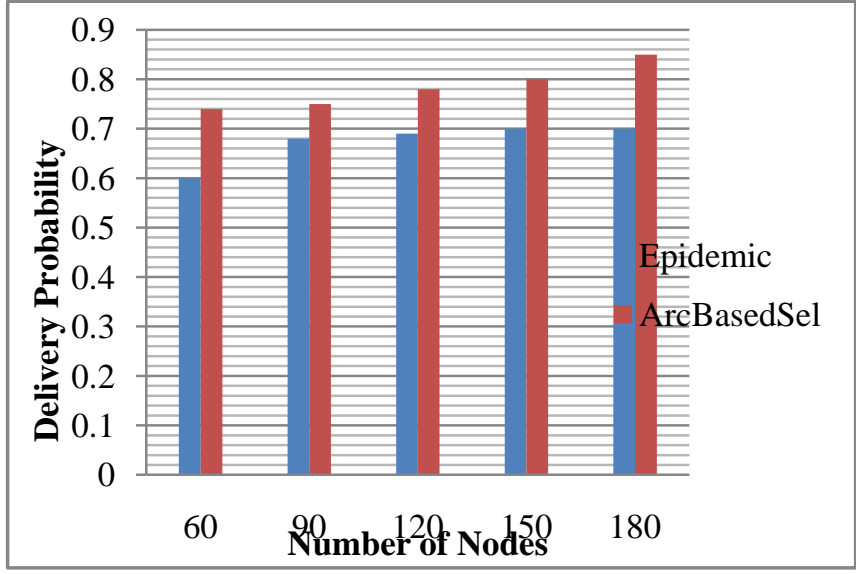

Figure 5. Delivery Probability vs Number of Nodes

Figure 5 depicts the effect of number of nodes on the delivery probability. It can be seen that when the number of nodes is varied, the average delivery probability of ArcBasedSel is $78.4 \%$ sec, which is better than obtained using Epidemic (which is $67.4 \%$ ). This is due to the reason as in ArcBasedSel, the selection of nodes is in the direction of destination. But in Epidemic, neighboring node may be in opposite direction also which lead to unreliable delivery of the message.

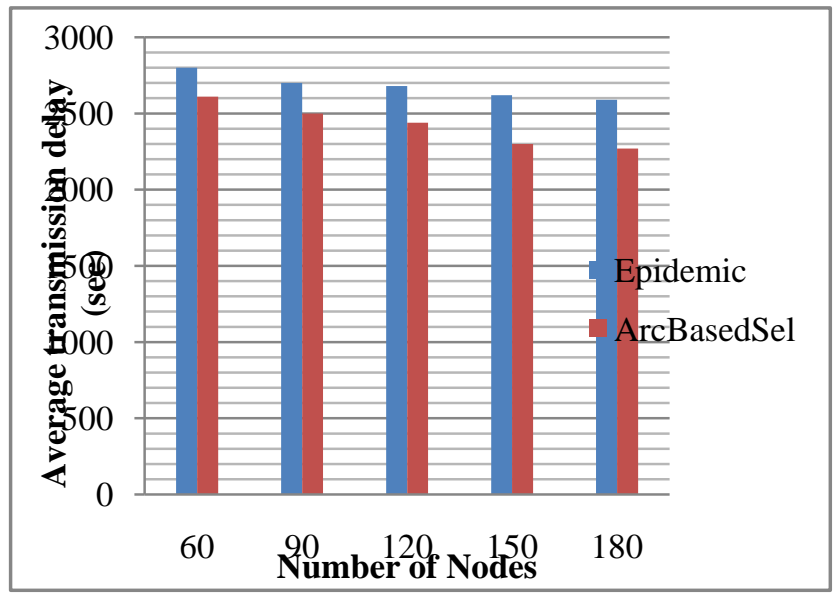

Figure 6. Average transmission delay vs Number of Nodes

In figure 6 , it can be observed that when the number of nodes varies, the mean average transmission delay of ArcBasedSel is $2424 \mathrm{sec}$, which is lesser than obtained using Epidemic (which is $2678 \mathrm{sec}$ ). This is due to the less number of intermediate nodes in routing path of ArcBasedSel. Hence the performance of ArcBasedSel is better than Epidemic.

\section{CONCLUSION AND FUTURE SCOPE}

In this paper, a work is proposed based on a directional arc selective flooding for opportunistic networks. By employing the directional arc, the flooding area is restricted in the direction of destination. This reduces the random flooding overheads in the network. Simulation results show that the proposed ArcBasedSel out-performs Epidemic in the overall performance of delivery, buffering overheads and average delay. Due to heterogeneous working nature of OppNets, an routing algorithm is needed that reaches the destination in less time with reliable delivery. In this case, the proposed ArcBasedSel is a good choice. In future, it will be compared with other OppNets protocols and also with more diverse routing parameters. 


\section{REFERENCES}

[1] K. Fall and S. Farrell, "DTN: an architectural retrospective," in IEEE Journal on Selected Areas in Communications, vol. 26, no. 5, pp. 828-836, June 2008. doi: 10.1109/JSAC.2008.080609

[2] C. M. Huang, K. c. Lan and C. Z. Tsai, "A Survey of Opportunistic Networks," 22nd International Conference on Advanced Information Networking and Applications Workshops (aina workshops 2008), Okinawa, 2008, pp. 1672-1677. doi: 10.1109/WAINA.2008.292

[3] S. Ali, J. Qadir and A. Baig, "Routing protocols in Delay Tolerant Networks - a survey," 2010 6th International Conference on Emerging Technologies (ICET), Islamabad, 2010, pp. 70-75. doi: 10.1109/ICET.2010.5638377

[4] http://ipnsig.org/

[5] P. Puri and M. P. Singh, "A survey paper on routing in delay-tolerant networks," 2013 International Conference on Information Systems and Computer Networks, Mathura, 2013, pp. 215-220.doi: 10.1109/ICISCON.2013.6524206

[6] Luming Wan, Feiyang Liu, Yawen Chen, and Haibo Zhang, " Routing Protocols for Delay Tolerant Networks: Survey and Performance Evaluation", International Journal of Wireless \& Mobile Networks (TJWMN) Vol. 7, No.3, June 2015
[7] S.Jain, K. Fall \& R. Patra, (2004). Routing in delay tolerant network, vol 34, no 4, pp 145-158, ACM.

[8] Spyropoulos, Thrasyvoulos, Konstantinos Psounis, and Cauligi S. Raghavendra,"Single-copy routing in intermittently connected mobile networks", First Annual IEEE Communications Society Conference on Sensor and AdHoc Communications and Networks, 2004.

[9] Vahdat, Amin, and David Becker. Epidemic routing for partially connected ad hoc networks. Technical Report CS-200006, Duke University, 2000.

[10] E. Jones, and p. A.s. Ward. "routing strategies for delaytolerant networks." submitted to acm computer communication review (ccr) (2006).

[11] T. Small, and Z. J. Haas. "Resource and performance tradeoffs in delay-tolerant wireless networks." Proceedings of the 2005 ACM SIGCOMM workshop on Delay-tolerant networking.ACM, 2005.

[12] T. Spyropoulos et al "Efficient routing in intermittently connected mobile networks: the multiple-copy case." networking, ieee/acm transactions on 16.1 (2008): 77-90.

[13] Kernen, Ari, Jrg Ott, and Teemu Krkkinen. "The ONE simulator for DTN protocol evaluation." Proceedings of the 2nd international conference on simulation tools and techniques. ICST (Institute for Computer Sciences, Social-Informatics and Telecommunications Engineering), 2009. 